\title{
EDITORIAL
}

\section{A neurosurgery resident's response to COVID-19: anything but routine}

\author{
Bryan D. Choi, MD, PhD \\ Department of Neurosurgery, Massachusetts General Hospital and Harvard Medical School, Boston, Massachusetts
}

$\mathrm{T}$ THE past weeks have been harrowing, as our world and individual communities grapple with the threat of COVID-19 to our health, productivity, and daily lives. As residents in neurosurgery we are doing everything we can to meet these challenges, by adhering to protocols that mitigate transmission during patient care, and supporting coworkers and staff, family and friends, while we all adjust to new realities.

At academic medical centers, a significant proportion of essential personnel consists of trainees. As I write, residents across the country have already been leading on the front lines, caring directly for patients infected with COVID-19. Because coronavirus primarily causes a respiratory illness, the first group of trainees minted by this pandemic will be our counterparts in emergency and internal medicine along with fellows in pulmonary and critical care. Neurosurgery, on the other hand, represents a smaller cadre, making up less than $0.5 \%$ of all physicians in the workforce. Given that distribution, how could neurosurgery residents contribute to the fight against COVID-19 in a meaningful way?

To help contextualize the experiences at our home institution, I connected with several colleagues in training across the country where COVID-19 was trending. The responses were resounding: "Everybody is stepping up to meet the crisis." In places like New York City, one of the US cities hardest hit by the COVID-19 epidemic, neurosurgery residents are already working overtime, in some cases pitching in to help with critically ill patients admitted to other services, and volunteering to support emergency screening clinics. All hands are on deck.

Having experienced upper respiratory symptoms in early March, I was among the first residents at our hospital to be tested for COVID-19. It took 72 hours of selfquarantine before my polymerase chain reaction (PCR) results came back negative. Later that week, I returned to a very different hospital than the one I had left; there was a palpable change, an air of uncertainty, and a relentless flow of protocols and recommendations supplied in every medium. This was followed by the introduction of several new policies including meticulous screening at hospital entrances and donning of masks at all times.

We would all ultimately find that neurosurgery training in the COVID-19 era was anything but routine. Departments throughout the nation have undertaken measures to ramp down surgical volume and redeploy skeleton inhouse call schedules to reduce the chances of cross-infection among house staff. Familiar duties like team rounds, assisting in surgical cases, and weekly conferences have been upended in favor of maneuvers to optimize social distancing and healthcare resource utilization, especially in cities where the impact has been most severe. And in those locations, like the San Francisco Bay Area, certain safety restrictions at the workplace have extended beyond the walls of the hospital, often in the form of shelter-inplace advisories, which have already been in effect for some time.

We are inspired as our fellow house staff navigate unfamiliar data, settings, and roles to accomplish what needs to be done. The usual cultivation of skills in the operating room has been supplanted by crash courses on minimizing the risk of exposure during neurosurgical procedures, such as those associated with aerosol generation from nasopharyngeal access or drilling of air sinuses. On the floor, the terms "post-op fever" and "chest pain" have taken on entirely new meanings, as responding clinicians seek to quickly assimilate nascent testing algorithms, triage workflows, and isolation guidelines. In an unprecedented fashion, department members have embraced web-based platforms to disseminate information rapidly and help counteract disruptions in resident education.

This pandemic will alter the tenets of our neurosurgical training, at least temporarily. After the dust settles, residents may look for guidance from our programs and governing bodies on how to most effectively manage the inevitable impact of coronavirus on case numbers, elective academic rotations, and, for some, the American Board of Neurological Surgery (ABNS) primary examination. Moreover, several trainees, many of whom may be competing for fellowships and junior faculty positions over the 
next year, will miss the chance to showcase their productivity at regularly scheduled annual meetings. Those expectations will all require recalibration.

As neurosurgery residents we are fortunate to have developed a particularly strong sense of community with each other, born in part out of a system that trains only a select few, with bonds that are forged during unique periods of stress and high acuity. We are a hardworking, clever, and independent group, but we also depend on each other a great deal, perhaps more than we realize. Unlike other fields with residencies that house more than a hundred trainees at a time, when a single member of our team is absent, we sense it immediately. Coronavirus-related policies as well as potential illnesses in our ranks put our esprit de corps at risk, leaving us both thankful for and challenged by the reminder of just how essential each member of our teams can be.

It is possible that well after this is published, the trajectory of COVID-19 will still be evolving, that consequences will be unforeseen and far-reaching, and that our training during the pandemic will continue to be anything but routine. But as neurosurgery residents we are tasked with sustaining the optimism that, as we set our sights on the horizon, there will also be countless opportunities to maintain vitality, to grow, and to keep learning, all the while supporting each other and those in our care.
Last, throughout this trying time we deeply thank our leadership and our institutions for making steadfast investments in our safety, advocating for personal protective equipment, providing transportation and care for our families, and adopting technologies that allow us to stay connected through virtual correspondence. No matter the challenges the coronavirus pandemic has yet to bring, with our combined efforts we can all still hope to emerge stronger together on the other side.

https://thejns.org/doi/abs/10.3171/2020.4.JNS201028

\section{Disclosures}

The author reports no conflict of interest.

\section{Correspondence}

Bryan D. Choi: bchoi1@partners.org.

INCLUDE WHEN CITING

Published online April 17, 2020; DOI: 10.3171/2020.4.JNS201028. 